\title{
HAUSDORFF DIMENSION AND PROJECTIONS RELATED TO INTERSECTIONS
}

\section{Mattila, Pertti}

2022

Mattila , P 2022 , ' HAUSDORFF DIMENSION AND PROJECTIONS RELATED TO

INTERSECTIONS ' , Publicacions matemàtiques , vol. 66 , no. 1 , pp. 305-323 . https://doi.org/10.5565/PUBLMAT66

http://hdl.handle.net/10138/340609

https://doi.org/10.5565/PUBLMAT6612212

unspecified

publishedVersion

Downloaded from Helda, University of Helsinki institutional repository.

This is an electronic reprint of the original article.

This reprint may differ from the original in pagination and typographic detail.

Please cite the original version. 
Publ. Mat. 66 (2022), 305-323

DOI: $10.5565 /$ PUBLMAT6612212

\title{
HAUSDORFF DIMENSION AND PROJECTIONS RELATED TO INTERSECTIONS
}

\author{
PertTi Mattila
}

\begin{abstract}
For $S_{g}(x, y)=x-g(y), x, y \in \mathbb{R}^{n}, g \in O(n)$, we investigate the Lebesgue measure and Hausdorff dimension of $S_{g}(A)$ given the dimension of $A$, both for general Borel subsets of $\mathbb{R}^{2 n}$ and for product sets.
\end{abstract}

2010 Mathematics Subject Classification: Primary: 28A75.

Key words: Hausdorff dimension, projection, intersection, Fourier transform.

\section{Introduction}

Let $A$ and $B$ be Borel subsets of $\mathbb{R}^{n}$. Under which conditions on the Hausdorff dimensions $\operatorname{dim} A$ and $\operatorname{dim} B$ do we have $A \cap(g(B)+z) \neq \emptyset$ for positively many, in the sense of Lebesgue measure $\mathcal{L}^{n}, z \in \mathbb{R}^{n}$ for almost all $g \in O(n)$ ? Defining $S_{g}(x, y)=x-g(y), A \cap(g(B)+z) \neq \emptyset$ for positively many $z \in \mathbb{R}^{n}$ is equivalent to $\mathcal{L}^{n}\left(S_{g}(A \times B)\right)>0$. Or we can also ask when $A \cap(g(B)+z) \neq \emptyset$ for $z$ in and $g$ outside a set of Hausdorff dimension of certain size. This reduces to estimating the dimension of $S_{g}(A \times B)$ and the dimension of the corresponding exceptional set of orthogonal transformations.

In this paper we study more generally the Lebesgue measure and Hausdorff dimension of $S_{g}(A)$ for $A \subset \mathbb{R}^{2 n}$. In Theorem 3.4 we shall show for a Borel set $A \subset \mathbb{R}^{2 n}$ that for almost all $g \in O(n), \mathcal{L}^{n}\left(S_{g}(A)\right)>0$, if $\operatorname{dim} A>n+1, \operatorname{dim} S_{g}(A) \geq \operatorname{dim} A-1$, if $n-1 \leq \operatorname{dim} A \leq n+1$, and $\operatorname{dim} S_{g}(A) \geq \operatorname{dim} A$, if $\operatorname{dim} A \leq n-1$. In all cases we also derive Hausdorff dimension estimates for the sets of exceptional $g \in O(n)$. In Theorems 4.2 and 4.3 we show that these estimates can be improved for product sets. We shall also comment on some relations to Falconer's distance set problem.

Instead of asking $A \cap(g(B)+z)$ to be non-empty, we could ask about the Hausdorff dimension of these intersections. This problem was studied in $[\mathbf{2 2}],[\mathbf{2 6}],[\mathbf{2 7}],[\mathbf{2 8}],[\mathbf{2 9}],[\mathbf{3 0}]$, and [31]. I shall make comments on it at the end of the paper. I expect the following to be true: if $A$ and $B$ are Borel subsets of $\mathbb{R}^{n}$ with $\operatorname{dim} A+\operatorname{dim} B>n$, then for almost all $g \in O(n)$, 
$\operatorname{dim} A \cap(g(B)+z) \geq \operatorname{dim} A+\operatorname{dim} B-n-\epsilon$ for every $\epsilon>0$ for positively many $z \in \mathbb{R}^{n}$. This is only known if one of the sets has dimension greater than $(n+1) / 2$. But in $[\mathbf{3 3}]$ we apply the results and methods of this paper to show that this is also true if $\operatorname{dim} A+(n-1) \operatorname{dim} B / n>n$ and $A$ and $B$ satisfy an extra condition of positive lower density.

The family $S_{g}, g \in O(n)$, is a restricted family of orthogonal projections onto $n$-planes in $\mathbb{R}^{2 n}$; it is only $n(n-1) / 2$-dimensional, while the full family of orthogonal projections has dimension $n^{2}$. Similar questions for other restricted families of orthogonal projections have been studied by many people; see $[\mathbf{1 7}],[\mathbf{1 6}],[\mathbf{1 1}],[\mathbf{3 6}],[\mathbf{3 4}],[\mathbf{3 5}],[\mathbf{2 1}],[\mathbf{3 7}]$. There are also discussions on these in $[\mathbf{3 0}]$ and $[\mathbf{3 2}]$.

Hausdorff dimension results for projections have their origin in Marstrand's projection theorem [25]: for a Borel set $A \subset \mathbb{R}^{2}$, for almost all orthogonal projections $p$ onto lines, $\mathcal{L}^{1}(p(A))>0$, if $\operatorname{dim} A>1$, and $\operatorname{dim} p(A)=\operatorname{dim} A$, if $\operatorname{dim} A \leq 1$. The study of exceptions was started by Kaufman [23], who showed that in the second statement the dimension of the set of the exceptional projections is at most $\operatorname{dim} A$, and continued by Falconer $[\mathbf{9}]$, who showed that in the first statement the set of the exceptions has dimension at most $2-\operatorname{dim} A$. Discussion and further references can be found, for example, in [30].

One could also study similar questions for other dimensions instead of the Hausdorff dimension, for instance, the Minkowski and packing dimensions. For these the situation is different. The examples given by Järvenpää in [18] show that there are sets with full dimension $n$ which project to measure zero on all $m$-planes. Hence the exact analogues of the intersection results of this paper are false even when one of the sets is a plane. However, Järvenpää in [19] and [20] and Eswarathasan, Iosevich, and Taylor in $[\mathbf{8}]$ proved some related results.

Acknowledgements. I would like to thank the referees for many useful comments.

\section{Preliminaries}

We denote by $\mathcal{L}^{n}$ the Lebesgue measure in the Euclidean $n$-space $\mathbb{R}^{n}$, $n \geq 2$, and by $\sigma^{n-1}$ the surface measure on the unit sphere $S^{n-1}$. The orthogonal group of $\mathbb{R}^{n}$ is $O(n)$ and its Haar probability measure is $\theta_{n}$. For $A \subset \mathbb{R}^{n}$ (or $A \subset O(n)$ ) we denote by $\mathcal{M}(A)$ the set of non-zero Radon measures $\mu$ on $\mathbb{R}^{n}$ with compact support spt $\mu \subset A$. The Fourier transform of $\mu$ is defined by

$$
\widehat{\mu}(x)=\int e^{-2 \pi i x \cdot y} d \mu y, \quad x \in \mathbb{R}^{n} .
$$

We shall also use $\mathcal{F}$ to denote the Fourier transform. 
For $0<s<n$ the $s$-energy of $\mu \in \mathcal{M}\left(\mathbb{R}^{n}\right)$ is

$$
I_{s}(\mu)=\iint|x-y|^{-s} d \mu x d \mu y=c(n, s) \int|\widehat{\mu}(x)|^{2}|x|^{s-n} d x .
$$

The second equality is a consequence of Parseval's formula and the fact that the distributional Fourier transform of the Riesz kernel $k_{s}, k_{s}(x)=$ $|x|^{-s}$, is a constant multiple of $k_{n-s}$; see, for example, [29, Lemma 12.12], or [30, Theorem 3.10]. These books contain most of the background material needed in this paper.

Notice that if $\mu$ satisfies the Frostman condition $\mu(B(x, r)) \leq r^{s}$ for all $x \in \mathbb{R}^{n}, r>0$, then $I_{t}(\mu)<\infty$ for all $t<s$. We have for any Borel set $A \subset \mathbb{R}^{n}$ with $\operatorname{dim} A>0$, cf. Theorem 8.9 in $[\mathbf{2 9}]$,

$$
\begin{aligned}
& \operatorname{dim} A=\sup \{s: \exists \mu \in \mathcal{M}(A) \text { such that } \\
&\left.\qquad \mu(B(x, r)) \leq r^{s} \text { for } x \in \mathbb{R}^{n}, r>0\right\} \\
&=\sup \left\{s: \exists \mu \in \mathcal{M}(A) \text { such that } I_{s}(\mu)<\infty\right\} .
\end{aligned}
$$

We shall denote by $f_{\#} \lambda$ the push-forward of a measure $\lambda$ under a map $f: f_{\#} \lambda(A)=\lambda\left(f^{-1}(A)\right)$.

By the notation $M \lesssim N$ we mean that $M \leq C N$ for some constant $C$. The dependence of $C$ should be clear from the context. The notation $M \approx N$ means that $M \lesssim N$ and $N \lesssim M$. By $c$ we mean positive constants with obvious dependence on the related parameters. The closed ball with centre $x$ and radius $r$ will be denoted by $B(x, r)$.

\section{Projections of general sets}

For $g \in O(n), t \in \mathbb{R}$, define

$$
S_{g}, \pi_{t}: \mathbb{R}^{n} \times \mathbb{R}^{n} \rightarrow \mathbb{R}^{n}, \quad S_{g}(x, y)=x-g(y), \quad \pi_{t}(x, y)=x-t y .
$$

Both of these can be realized as families of orthogonal projections. The family $S_{g}$ has curvature (in any natural sense), while $\pi_{t}$ does not. See $[\mathbf{1 1}],[\mathbf{3 6}]$, and $[\mathbf{2 1}]$ for the role of curvature in projection theorems.

More precisely, let $\left\{e_{1}, \ldots, e_{n}\right\}$ be an orthonormal basis for $\mathbb{R}^{n}$. Set $u_{i}=\frac{1}{\sqrt{2}}\left(e_{i},-g^{-1}\left(e_{i}\right)\right), i=1, \ldots, n$. Then $\left\{u_{1}, \ldots, u_{n}\right\}$ is an orthonormal basis for an $n$-plane $V_{g} \subset \mathbb{R}^{2 n}$. The orthogonal complement of $V_{g}$, spanned by $\frac{1}{\sqrt{2}}\left(e_{i}, g^{-1}\left(e_{i}\right)\right), i=1, \ldots, n$, is the kernel of $S_{g}$. Since $\frac{1}{\sqrt{2}} S_{g}\left(u_{i}\right)=e_{i}, \frac{1}{\sqrt{2}} S_{g}$ is essentially the orthogonal projection onto $V_{g}$.

When $n=2$ we have, in complex notation, $g$ identified with the angle $\phi: S_{g}(x, y)=x-e^{i \phi} y$.

Some relations between the projections $\pi_{t}$ and the Kakeya problem are discussed in $[\mathbf{3 2}]$. 
Recall the following lemma from [31, Lemma 2.1]. In [31] only the first bound was proven, but the second can be proven by an analogous argument to that used for the first bound. Notice that the term $(n-$ 1) $(n-2) / 2$ is needed there: the subgroup of $O(n)$ consisting of $(x, t) \mapsto$ $(g(x), t), x \in \mathbb{R}^{n-1}, t \in \mathbb{R}, g \in O(n-1)$, has dimension $(n-1)(n-2) / 2$ and $(g(0), 1)=(0,1)$ for all $g \in O(n-1)$.

Lemma 3.1. Let $\theta \in \mathcal{M}(O(n)), \alpha>(n-1)(n-2) / 2$, and $\beta=\alpha-$ $(n-1)(n-2) / 2$. If $\theta(B(g, r)) \leq r^{\alpha}$ for all $g \in O(n)$ and $r>0$, then for $x, z \in \mathbb{R}^{n} \backslash\{0\}, r>0$,

$$
\theta(\{g:|x-g(z)|<r\}) \lesssim \min \left\{(r /|z|)^{\beta},(r /|x|)^{\beta}\right\} .
$$

This will be applied via the following proposition, as in Chapter 5 of $[\mathbf{3 0}]$ and in many other places:

Proposition 3.2. Let $A \subset \mathbb{R}^{n}$ be a Borel set and $\beta>0, \gamma>0$. Suppose that for any $\theta \in \mathcal{M}(O(n))$ such that (3.1) holds, $\mathcal{L}^{n}\left(S_{g}(A)\right)>0$ (or $\left.\operatorname{dim} S_{g}(A) \geq \gamma\right)$ for $\theta$ almost all $g \in O(n)$. Then there is a Borel set $E \subset$ $O(n)$ such that $\operatorname{dim} E \leq \beta+(n-1)(n-2) / 2$ and $\mathcal{L}^{n}\left(S_{g}(A)\right)>0$ (or $\left.\operatorname{dim} S_{g}(A) \geq \gamma\right)$ for $g \in O(n) \backslash E$.

Proof: I skip the easy measurability arguments. If the Lebesgue measure part fails, the set $G$ of $g \in O(n)$ for which $\mathcal{L}^{n}\left(S_{g}(A)\right)=0$ has dimension greater than $\alpha=\beta+(n-1)(n-2) / 2$. Then by (2.2) there is $\theta \in \mathcal{M}(G)$ such that $\theta(B(g, r)) \leq r^{\alpha}$ for all $g \in O(n)$ and $r>0$, so that (3.1) holds by Lemma 3.1. By assumption, $\mathcal{L}^{n}\left(S_{g}(A)\right)>0$ for $\theta$ almost all $g \in O(n)$, which contradicts the definition $G$ and that $\theta \in \mathcal{M}(G)$. The Hausdorff dimension part is proven by the same argument.

The following theorem for $\pi_{t}$ is essentially a special case of Oberlin's results in [34]. It was not explicitly stated there, but (1) and (2) follow by his arguments; see in particular the proof of Lemma 3.1 in [34]. The proof of (3) is a standard argument of Kaufman from [23]; see the proof of Theorem 3.4. The proof of Theorem 3.4 also gives Theorem 3.3, changing $g(x)$ to $t x$.

Theorem 3.3. Let $A \subset \mathbb{R}^{2 n}$ be a Borel set.

(1) If $\operatorname{dim} A>2 n-1$, then $\mathcal{L}^{n}\left(\pi_{t}(A)\right)>0$ for $\mathcal{L}^{1}$ almost all $t \in \mathbb{R}$. Moreover, there is $E \subset \mathbb{R}$ such that $\operatorname{dim} E \leq 2 n-\operatorname{dim} A$ and $\mathcal{L}^{n}\left(\pi_{t}(A)\right)>0$ for $t \in \mathbb{R} \backslash E$.

(2) If $n \leq \operatorname{dim} A \leq 2 n-1$, then $\operatorname{dim} \pi_{t}(A) \geq \operatorname{dim} A-n+1$ for $\mathcal{L}^{1}$ almost all $t \in \mathbb{R}$. Moreover, for $\operatorname{dim} A-n \leq u \leq \operatorname{dim} A-n+1$ there is $E \subset \mathbb{R}$ such that $\operatorname{dim} E \leq u+n-\operatorname{dim} A$ and $\operatorname{dim} \pi_{t}(A) \geq u$ for $t \in \mathbb{R} \backslash E$. 
(3) If $\operatorname{dim} A \leq n$, then $\operatorname{dim} \pi_{t}(A) \geq \min \{\operatorname{dim} A, 1\}$ for $\mathcal{L}^{1}$ almost all $t \in \mathbb{R}$. Moreover, for $0<u \leq \min \{\operatorname{dim} A, 1\}$ there is $E \subset \mathbb{R}$ such that $\operatorname{dim} E \leq u$ and $\operatorname{dim} \pi_{t}(A) \geq u$ for $t \in \mathbb{R} \backslash E$.

(4) For all $t \in \mathbb{R}, \operatorname{dim} \pi_{t}(A) \geq \operatorname{dim} A-n$.

Notice that the last statement is trivial, because associating with $\pi_{t}$ an orthogonal projection $p_{t}$, as for $S_{g}$ at the beginning of this section, $A \subset$ $p_{t}(A) \times p_{t}^{-1}(0)$ and $\operatorname{dim}\left(p_{t}(A) \times p_{t}^{-1}(0)\right)=\operatorname{dim} p_{t}(A)+n=\operatorname{dim} \pi_{t}(A)+n$.

This theorem is valid also when $n=1$; it is Marstrand's projection with Kaufman's and Falconer's exceptional set estimates.

We have a similar result for $S_{g}$. Observe also there that (4) is trivial. The proof below for (1) and (2) is a modification of Oberlin's proof. The proof of (3) again is Kaufman's argument.

Theorem 3.4. Let $A \subset \mathbb{R}^{2 n}$ be a Borel set.

(1) If $\operatorname{dim} A>n+1$, then $\mathcal{L}^{n}\left(S_{g}(A)\right)>0$ for $\theta_{n}$ almost all $g \in O(n)$. Moreover, there is $E \subset O(n)$ such that $\operatorname{dim} E \leq 2 n-\operatorname{dim} A+(n-$ 1) $(n-2) / 2$ and $\mathcal{L}^{n}\left(S_{g}(A)\right)>0$ for $g \in O(n) \backslash E$.

(2) If $n-1 \leq \operatorname{dim} A \leq n+1$, then $\operatorname{dim} S_{g}(A) \geq \operatorname{dim} A-1$ for $\theta_{n}$ almost all $g \in O(n)$. Moreover, for any $\operatorname{dim} A-n \leq u \leq \operatorname{dim} A-1$ there is $E \subset O(n)$ such that $\operatorname{dim} E \leq u+n-\operatorname{dim} A+(n-1)(n-2) / 2$ and $\operatorname{dim} S_{g}(A) \geq u$ for $g \in O(n) \backslash E$.

(3) If $\operatorname{dim} A \leq n-1$, then $\operatorname{dim} S_{g}(A) \geq \operatorname{dim} A$ for $\theta_{n}$ almost all $g \in$ $O(n)$. Moreover, for $0<u \leq \operatorname{dim} A$ there is $E \subset O(n)$ such that $\operatorname{dim} E \leq u+(n-1)(n-2) / 2$ and $\operatorname{dim} S_{g}(A) \geq u$ for $g \in O(n) \backslash E$.

(4) For all $g \in O(n)$, $\operatorname{dim} S_{g}(A) \geq \operatorname{dim} A-n$.

Proof: Let $0<s<\operatorname{dim} A$ and $\mu \in \mathcal{M}(A)$ with $I_{s}(\mu)<\infty$. $\mathbb{R}^{n}$

Let $\mu_{g} \in \mathcal{M}\left(S_{g}(A)\right)$ be the push-forward of $\mu$ under $S_{g}$. Then for $\xi \in$

$$
\begin{aligned}
\widehat{\mu_{g}}(\xi) & =\int e^{-2 \pi i \xi \cdot S_{g}(x, y)} d \mu(x, y) \\
& =\int e^{-2 \pi i\left(\xi,-g^{-1}(\xi)\right) \cdot(x, y)} d \mu(x, y)=\widehat{\mu}\left(\xi,-g^{-1}(\xi)\right) .
\end{aligned}
$$

Let $0<\beta \leq n-1$ and let $\theta \in \mathcal{M}(O(n))$ be such that for $x, z \in \mathbb{R}^{n} \backslash\{0\}$, $r>0$,

$$
\theta(\{g \in O(n):|x-g(z)|<r\}) \leq \min \left\{(r /|z|)^{\beta},(r /|x|)^{\beta}\right\} .
$$

To prove (1) and (2) we shall show that for $R>1$

$$
\iint_{R \leq|\xi| \leq 2 R}\left|\widehat{\mu}\left(\xi,-g^{-1}(\xi)\right)\right|^{2} d \xi d \theta g \lesssim R^{2 n-s-\beta} .
$$


This is applied to the dyadic annuli, $R=2^{k}, k=1,2, \ldots$ The sum converges if $s>2 n-\beta$, and we can choose $\mu$ with such $s$ if $\operatorname{dim} A>2 n-\beta$. This gives $\iint\left|\widehat{\mu_{g}}(\xi)\right|^{2} d \xi d \theta g<\infty$. Hence for $\theta$ almost all $g \in O(n), \mu_{g}$ is absolutely continuous with $L^{2}$ density, and so $\mathcal{L}^{n}\left(S_{g}(A)\right)>0$. Taking $\beta=n-1$ and $\theta=\theta_{n}$, we get the first part of (1). The second follows with general $\beta$ and $\theta$ using Proposition 3.2.

To prove part (2) let $0<u<s+\beta-n$ and $\mu$ as above. Then (3.3) yields

$$
\iint\left|\widehat{\mu_{g}}(\xi)\right|^{2}|\xi|^{u-n} d \xi d \theta g<\infty
$$

so by (2.1) and (2.2), $\operatorname{dim} S_{g}(A) \geq u$ for $\theta$ almost all $g \in O(n)$ and thus (2) follows with the same argument as above.

Now we begin the proof of (3.3). From (3.2) we get for $\xi, y \in \mathbb{R}^{n}$, $R \leq|\xi| \leq 2 R, M>\beta$,

$$
\int(1+|\xi+g(y)|)^{-M} d \theta g \lesssim R^{-\beta}
$$

because

$$
\begin{aligned}
& \int(1+|\xi+g(y)|)^{-M} d \theta g \\
& \quad \leq \theta(\{g \in O(n):|\xi+g(y)|<1\})+\int_{\{g:|\xi+g(y)| \geq 1\}}(1+|\xi+g(y)|)^{-M} d \theta g \\
& \quad \lesssim R^{-\beta}+\sum_{j=0}^{\infty} 2^{-M j} \theta\left(\left\{g \in O(n): 2^{j} \leq|\xi+g(y)|<2^{j+1}\right\}\right) \\
& \lesssim R^{-\beta}+\sum_{j=0}^{\infty} 2^{-M j}\left(2^{j} /|\xi|\right)^{\beta} \lesssim R^{-\beta} .
\end{aligned}
$$

Choose a smooth compactly supported function $\phi$ which equals 1 on the support of $\mu$. Then $\widehat{\mu}=\widehat{\phi \mu}=\widehat{\phi} * \widehat{\mu}$ and the integral in (3.3) equals

$$
\begin{aligned}
I_{R} & :=\iint_{R \leq|\xi| \leq 2 R}\left|\widehat{\phi \mu}\left(\xi,-g^{-1}(\xi)\right)\right|^{2} d \xi d \theta g \\
& =\iint_{R \leq|\xi| \leq 2 R}\left|\int \widehat{\phi}\left(\left(\xi,-g^{-1}(\xi)\right)-y\right) \widehat{\mu}(y) d y\right|^{2} d \xi d \theta g .
\end{aligned}
$$


By the Schwartz inequality,

$$
\begin{aligned}
I_{R} \leq \iint_{R \leq|\xi| \leq 2 R}\left(\int\left|\widehat{\phi}\left(\left(\xi,-g^{-1}(\xi)\right)-y\right)\right| d y\right. \\
\left.\quad \times \int\left|\widehat{\phi}\left(\left(\xi,-g^{-1}(\xi)\right)-y\right)\right||\widehat{\mu}(y)|^{2} d y\right) d \xi d \theta g \\
\quad \lesssim \iint_{R \leq|\xi| \leq 2 R} \int\left|\widehat{\phi}\left(\left(\xi,-g^{-1}(\xi)\right)-y\right)\right||\widehat{\mu}(y)|^{2} d y d \xi d \theta g \\
\leq \iint_{R \leq|\xi| \leq 2 R} \int\left(1+\left|\left(\xi,-g^{-1}(\xi)\right)-y\right|\right)^{-3 M}|\widehat{\mu}(y)|^{2} d y d \xi d \theta g
\end{aligned}
$$

by the fast decay of $\widehat{\phi}$, where $M>2 n$. Clearly, with $y=\left(y_{1}, y_{2}\right), y_{1}, y_{2} \in$ $\mathbb{R}^{n}$,

$$
\left.\mid\left(\xi,-g^{-1}(\xi)\right)-y\right) \mid \geq \max \left\{\left|\xi-y_{1}\right|,\left|\xi+g\left(y_{2}\right)\right|\right\} .
$$

Moreover, $\left.\mid\left(\xi,-g^{-1}(\xi)\right)-y\right)|\approx| y \mid$, when $R \leq|\xi| \leq 2 R$ and $|y|>5 R$. Hence

$$
\begin{aligned}
I_{R} \lesssim \int_{|y| \leq 5 R} \int_{R \leq|\xi| \leq 2 R} \int\left(1+\left|\xi+g\left(y_{2}\right)\right|\right)^{-M} d \theta \\
\times g\left(1+\left|\xi-y_{1}\right|\right)^{-M} d \xi|\widehat{\mu}(y)|^{2} d y \\
+\int_{|y|>5 R} \int_{R \leq|\xi| \leq 2 R} \int\left(1+\left|\xi+g\left(y_{2}\right)\right|\right)^{-M} d \theta \\
\times g\left(1+\left|\xi-y_{1}\right|\right)^{-M} d \xi|y|^{-M} d y .
\end{aligned}
$$

We have by (3.4)

$$
\int\left(1+\left|\xi+g\left(y_{2}\right)\right|\right)^{-M} d \theta g \lesssim R^{-\beta}
$$

Since $\int\left(1+\left|\xi-y_{1}\right|\right)^{-M} d \xi$ is bounded, we obtain

$$
I_{R} \lesssim R^{-\beta}\left(\int_{|y| \leq 5 R}|\widehat{\mu}(y)|^{2} d y+\int_{|y|>5 R}|y|^{-M} d y\right) .
$$

The second integral is bounded and for the first we have by $[\mathbf{3 0}$, Section 3.8],

$$
\int_{|y| \leq 5 R}|\widehat{\mu}(y)|^{2} d y \lesssim R^{2 n-s} .
$$

This implies $I_{R} \lesssim R^{2 n-s-\beta}$ as required. Thus we have proven (3.3), hence also (1) and (2).

To prove (3) suppose that $0<u<\operatorname{dim} A \leq n-1, \mu \in \mathcal{M}(A)$ with $I_{u}(\mu)<\infty$, and let $\theta$ and $\beta$ be as in (3.2) with $\beta>u$. It suffices to show 
that $\operatorname{dim} S_{g}(A) \geq u$ for $\theta$ almost all $g \in O(n)$. Using (3.2) this follows from

$$
\begin{aligned}
\int I_{u}\left(\mu_{g}\right) d \theta g= & \iiint|x-y|^{-u} d S_{g \#} \mu x d S_{g \#} \mu y d \theta g \\
= & \iiint\left|S_{g}(w-z)\right|^{-u} d \mu w d \mu z d \theta g \\
= & \iiint_{0}^{\infty} \theta\left(\left\{g:\left|S_{g}(w-z)\right|^{-u}>r\right\}\right) d r d \mu w d \mu z \\
= & \iiint_{0}^{\infty} \theta\left(\left\{g:\left|S_{g}(w-z)\right|<r^{-1 / u}\right\}\right) d r d \mu w d \mu z \\
\lesssim & \iiint_{0}^{|w-z|^{-u}} d r d \mu w d \mu z \\
& +\iiint_{|w-z|^{-u}}^{\infty}\left(r^{-1 / u} /|w-z|\right)^{\beta} d r d \mu w d \mu z \\
\approx & I_{u}(\mu)<\infty,
\end{aligned}
$$

which completes the proof of the theorem.

3.1. Sharpness. The bounds in the $\mathcal{L}^{1}$ almost all statements of Theorem 3.3 are sharp when $n=2$. To see this let $0 \leq s \leq 1, C_{s} \subset \mathbb{R}$ with $\operatorname{dim} C_{s}=s$, and $A_{s}=\left\{(x, y) \in \mathbb{R}^{2} \times \mathbb{R}^{2}: x_{1} \in C_{s}, y_{1}=0\right\}$. Then $\operatorname{dim} A_{s}=2+s, \pi_{t}\left(A_{s}\right)=C_{s} \times \mathbb{R}$, and $\operatorname{dim} \pi_{t}\left(A_{s}\right)=1+s$. This shows that (2) is sharp. For (1) we can choose $C_{1}$ with $\mathcal{L}^{1}\left(C_{1}\right)=0$, then $\operatorname{dim} A_{1}=3$ and $\mathcal{L}^{2}\left(\pi_{t}(A)\right)=0$. If $1 \leq \operatorname{dim} A \leq 2$, we can only say that $\operatorname{dim} \pi_{t}(A) \geq 1$ for almost all $t \in \mathbb{R}$ since $\pi_{t}(\mathbb{R} \times\{0\} \times \mathbb{R} \times\{0\})=\mathbb{R} \times\{0\}$. Hence (3) is also sharp. Probably the bounds for the dimensions of the exceptional sets are sharp, too. Perhaps this could be seen using similar examples as in [24]; see also Example 5.13 in [30], but I have not checked it.

When $n \geq 3$ a similar argument shows that the $\mathcal{L}^{1}$ almost all statements of Theorem 3.3 are sharp when $\operatorname{dim} A \geq 2 n-2$ or $\operatorname{dim} A \leq 2$. Probably it is not sharp in the remaining ranges.

Harris has shown in [14] that the bounds in Theorem 3.4 are sharp. In Section 4 we shall see that some of them can be improved for product sets. By the above examples this is not possible for $\pi_{t}$.

I illustrate the role of $\operatorname{dim} O(n-1)=(n-1)(n-2) / 2$ with two simple examples: For $0<s \leq 1$ choose a compact set $C_{s} \subset \mathbb{R}$ such that $\operatorname{dim} C_{s}=\operatorname{dim}\left(C_{s}-C_{s}\right)=s, \operatorname{dim}\left(C_{s} \times C_{s}\right)=2 s$, and $\mathcal{L}^{1}\left(C_{1}-C_{1}\right)=0$. Such sets are easy to construct. If $A_{s}=\mathbb{R}^{n-1} \times C_{s} \times \mathbb{R}^{n-1} \times C_{s}$, then $\operatorname{dim} A_{s}=2 s+2(n-1), S_{g}(A)=\mathbb{R}^{n-1} \times\left(C_{s}-C_{s}\right)$, and $\operatorname{dim} S_{g}\left(A_{s}\right)=$ 
$s+n-1$ for $g \in O(n-1)$ (identified with $(x, t) \mapsto(g(x), t))$. In particular, $\operatorname{dim} A_{1}=2 n$ and $\mathcal{L}^{n}\left(S_{g}\left(A_{1}\right)\right)=0$ for $g \in O(n-1)$. Next, take $B_{s}=$ $\{0\} \times C_{s} \times\{0\} \times C_{s}$. Then $\operatorname{dim} B_{s}=2 s, S_{g}(A)=\{0\} \times\left(C_{s}-C_{s}\right)$, and $\operatorname{dim} S_{g}\left(B_{s}\right)=s$ for $g \in O(n-1)$.

3.2. An alternative argument. Here is another simple argument for the statement 'If $A \subset \mathbb{R}^{2 n}$ is a Borel set and $\operatorname{dim} A>n+1$, then $\mathcal{L}^{n}\left(S_{g}(A)\right)>0$ for $\theta_{n}$ almost all $g \in O(n)$ ':

Let $\mu \in \mathcal{M}(A)$ with $I_{n+1}(\mu)<\infty$. Consider for $r>0$

$$
\begin{aligned}
I_{r} & =r^{-n} \iint S_{g \# \mu}(B(z, r)) d S_{g \#} \mu z d \theta_{n} g \\
& =r^{-n} \iint \theta_{n}(\{g:|x-u-g(y-v)| \leq r\}) d \mu(u, v) d \mu(x, y) \\
& \lesssim r^{-1} \iint_{\{(x, y):|| x-u|-| y-v|| \leq r\}}|y-v|^{1-n} d \mu(u, v) d \mu(x, y) .
\end{aligned}
$$

Let $\phi \in C_{0}^{\infty}\left(\mathbb{R}^{n}\right)$ with $\phi(y)=1$ when $(x, y) \in \operatorname{spt} \mu$ for some $x$, and let

$$
\psi_{r}(x, y)=\chi_{\{(x, y):|| x|-| y|| \leq r\}}(x, y)|y|^{1-n} \phi(y) .
$$

Then

$$
I_{r} \lesssim r^{-1} \int \psi_{r} * \mu d \mu=r^{-1} \int \widehat{\psi_{r}}|\widehat{\mu}|^{2} .
$$

Let $\sigma_{r}$ be the surface measure on $\left\{x \in \mathbb{R}^{n}:|x|=r\right\}$. Then for any $u, y \in$ $\mathbb{R}^{n}, \widehat{\sigma_{|y|}}(u)=|y|^{n-1}|u|^{1-n} \widehat{\sigma_{|u|}}(y)$. Thus for small $r$

$$
\begin{aligned}
\left|r^{-1} \widehat{\psi_{r}}(u, v)\right| & =\left.\left|r^{-1} \iint_{|| x|-| y|| \leq r}\right| y\right|^{1-n} \phi(y) e^{-2 \pi i(u \cdot x+v \cdot y)} d x d y \mid \\
& \left.\approx\left|\int\right| y\right|^{1-n} \phi(y) \widehat{\sigma_{|y|}}(u) e^{-2 \pi i v \cdot y} d y \mid \\
& =\left.\left|\int\right| y\right|^{1-n} \phi(y)|y|^{n-1}|u|^{1-n} \widehat{\sigma_{|u|}}(y) e^{-2 \pi i v \cdot y} d y \mid \\
& =\left.|| u\right|^{1-n} \mathcal{F}\left(\phi \widehat{\sigma_{|u|}}\right)(v)|=||u|^{1-n} \int \widehat{\phi}(y-v) d \sigma_{|u|} y \mid \\
& \lesssim|u|^{1-n}(1+|| u|-| v||)^{1-n} \lesssim|(u, v)|^{1-n},
\end{aligned}
$$

the second to last by the fast decay of $\widehat{\phi}$. Hence

$$
I_{r} \lesssim \iint|(u, v)|^{1-n}|\widehat{\mu}(u, v)|^{2} d(u, v) \approx I_{n+1}(\mu) .
$$


Define the lower derivative, with $\alpha(n)=\mathcal{L}^{n}(B(0,1))$,

$$
\underline{D}\left(S_{g \#} \mu\right)(z)=\liminf _{r \rightarrow 0} \alpha(n)^{-1} r^{-n} S_{g \#} \mu(B(z, r)) .
$$

Letting $r \rightarrow 0$ and using Fatou's lemma we then see that

$$
\iint \underline{D}\left(S_{g \#}\right)(z)^{2} d z d \theta_{n} g=\int \underline{D}\left(S_{g \#}\right)(z) d S_{g \#} \mu z d \theta_{n} g<\infty,
$$

which implies that $S_{g \#} \mu \ll \mathcal{L}^{n}$ with $L^{2}$ density; see e.g. [29, Theorem 2.12], for $\theta_{n}$ almost all $g$, from which the claim follows.

\section{Product sets}

For product sets we can improve Theorem 3.4 for $S_{g}$, but not for $\pi_{t}$, as the previous examples show. Let $\theta \in \mathcal{M}\left(S^{n-1}\right)$ and $0<\beta \leq n-1$. Suppose that for $x, z \in \mathbb{R}^{n}, r>1$,

$$
\theta(\{g:|x-g(z)|<r\}) \lesssim(r /|x|)^{\beta} .
$$

Let $\mu \in \mathcal{M}\left(\mathbb{R}^{n}\right)$ and set for $r>1$ and $\xi \in \mathbb{R}^{n}$

$$
\begin{aligned}
\sigma(\mu)(r) & =\int_{S^{n-1}}|\widehat{\mu}(r v)|^{2} d \sigma^{n-1} v, \\
\sigma_{\theta}(\mu)(\xi) & =\int\left|\widehat{\mu}\left(g^{-1}(\xi)\right)\right|^{2} d \theta g .
\end{aligned}
$$

Then $\sigma_{\theta_{n}}(\mu)(\xi)=c \sigma(\mu)(|\xi|)$.

The decay estimates for $\sigma(\mu)(r)$ have been studied by many people; a discussion can be found in $[\mathbf{3 0}]$. The best-known estimates, due to Wolff [38], when $n=2$, and to Du and Zhang [6], in the general case, are the following: Let $\mu \in \mathcal{M}\left(\mathbb{R}^{n}\right)$ with $\mu(B(x, r)) \leq r^{s}$ for $x \in \mathbb{R}^{n}$, $r>0$. Then for all $\epsilon>0, r>1$,

$$
\sigma(\mu)(r) \lesssim \begin{cases}r^{-(n-1) s / n+\epsilon} & \text { for all } 0<s<n \\ r^{-(n-1) / 2+\epsilon} & \text { if } 0<(n-1) / 2 \leq s \leq n / 2, \\ r^{-s+\epsilon} & \text { if } 0<s \leq(n-1) / 2 .\end{cases}
$$

The middle estimate is rather unnecessary to state since it follows from the last. I shall drop the corresponding case later on. The essential case for the first estimate is $s>n / 2$, otherwise the second and third estimates are better. Up to $\epsilon$ these estimates are sharp when $n=2$. When $n \geq 3$ the sharp bounds are not known for all $s$; see $[\mathbf{3}]$ for discussion and the most recent examples.

For $r>1$, let

$$
A_{r}=\left\{x \in \mathbb{R}^{n}: r-1<|x|<r+1\right\} .
$$


It is easy to see that for large $r, \sigma(\mu)(r) \lesssim r^{-\alpha+\epsilon}$ for all $\epsilon>0$ if and only if $r^{1-n} \int_{A_{r}}|\widehat{\mu}(x)|^{2} d x \lesssim r^{-\alpha+\epsilon}$ for all $\epsilon>0$. Indeed, the implication from left to right is trivial. The opposite implication is Proposition 16.2 in [30]. This and the above estimates for $\sigma(\mu)(r)$ yield the following lemma:

Lemma 4.1. Let $\theta \in \mathcal{M}\left(S^{n-1}\right)$ satisfy (4.1) with exponent $0<\beta \leq$ $n-1$. If $\mu(B(x, r)) \leq r^{s}$ for $x \in \mathbb{R}^{n}, r>0$, then for every $\xi \in \mathbb{R}^{n}$ with $|\xi|>1$ and for every $\epsilon>0$

$$
\sigma_{\theta}(\mu)(\xi) \lesssim \begin{cases}|\xi|^{-(n-1) s / n+n-1-\beta+\epsilon} & \text { for all } 0<s<n, \\ |\xi|^{-s+n-1-\beta+\epsilon} & \text { if } 0<s \leq(n-1) / 2 .\end{cases}
$$

Proof: I only consider the first estimate; the second follows in the same way. Using the above estimate for $\sigma(\mu)(r)$ and the above mentioned relation to the estimates over the annuli $A_{r}$, we have

$$
r^{1-n} \int_{A_{r}}|\widehat{\mu}(x)|^{2} d x \lesssim r^{-(n-1) s / n+\epsilon}
$$

for all $\epsilon>0$. The proof of Proposition 16.2 in [30] works for $\theta$ instead of $\theta_{n}$ as such, yielding the required estimate. I give a short sketch.

Choose a smooth function $\varphi, \varphi \geq 0$, such that $\varphi=1$ on spt $\mu$. Then arguments similar to those in the proof of Theorem 3.4 show that

$$
\begin{aligned}
& \sigma_{\theta}(\mu)(\xi) \lesssim \iint_{\left\{(x, g):\left|g^{-1}(\xi)-x\right|<|\xi|^{\varepsilon}\right\}} d \theta g|\widehat{\mu}(x)|^{2} d x \\
+ & \sum_{j=1}^{\infty} \iint_{\left\{(x, g):\left(|\xi|^{\varepsilon}\right)^{j} \leq\left|g^{-1}(\xi)-x\right|<\left(|\xi|^{\varepsilon}\right)^{j+1}\right\}}\left|\widehat{\varphi}\left(g^{-1}(\xi)-x\right)\right||\widehat{\mu}(x)|^{2} d x d \theta g .
\end{aligned}
$$

The second term is easily handled by (4.3) and the fast decay of $\varphi$.

We have by (4.1)

$$
\theta\left(\left\{g:\left|g^{-1}(\xi)-x\right|<|\xi|^{\varepsilon}\right\}\right) \lesssim|\xi|^{(\varepsilon-1) \beta},
$$

whence the first term is bounded by

$$
|\xi|^{(\varepsilon-1) \beta} \int_{\left\{x:|\xi|-|\xi|^{\varepsilon}<|x|<|\xi|+|\xi|^{\varepsilon}\right\}}|\widehat{\mu}(x)|^{2} d x .
$$

Covering the interval $\left(|\xi|-|\xi|^{\varepsilon},|\xi|+|\xi|^{\varepsilon}\right)$ with about $|\xi|^{\varepsilon}$ intervals of length 2 and applying (4.3) we obtain the required bound. 
Theorem 4.2. Let $A, B \subset \mathbb{R}^{n}$ be Borel sets.

(1) Suppose $\operatorname{dim} A+\operatorname{dim} B>n$. If $\operatorname{dim} A+(n-1) \operatorname{dim} B / n>n$ or $\operatorname{dim} A>(n+1) / 2$, then $\mathcal{L}^{2}\left(S_{g}(A \times B)\right)>0$ for $\theta_{n}$ almost all $g \in O(n)$.

(2) If $\operatorname{dim} A+(n-1) \operatorname{dim} B / n \leq n$, then $\operatorname{dim} S_{g}(A \times B) \geq \operatorname{dim} A+$ $(n-1) \operatorname{dim} B / n$ for $\theta_{n}$ almost all $g \in O(n)$.

If $\operatorname{dim} A+\operatorname{dim} B \leq n$ and $\operatorname{dim} B \leq(n-1) / 2$, then $\operatorname{dim} S_{g}(A \times B) \geq$ $\operatorname{dim} A+\operatorname{dim} B$ for $\theta_{n}$ almost all $g \in O(n)$.

We have the following exceptional set estimates:

Theorem 4.3. Let $A, B \subset \mathbb{R}^{n}$ be Borel sets.

(1) Suppose $\operatorname{dim} A+\operatorname{dim} B>n$. Then there is $E \subset O(n)$ such that $\mathcal{L}^{2}\left(S_{g}(A \times B)\right)>0$ for $g \in O(n) \backslash E$ and $\operatorname{dim} E \leq 2 n-1-$ $\operatorname{dim} A-(n-1) \operatorname{dim} B / n+(n-1)(n-2) / 2$. Moreover, $\operatorname{dim} E \leq$ $2 n-1-\operatorname{dim} A-\operatorname{dim} B+(n-1)(n-2) / 2$, if $\operatorname{dim} B \leq(n-1) / 2$.

(2) Let $0<\alpha \leq n$. Then there is $E \subset O(n)$ such that $\operatorname{dim} S_{g}(A \times B) \geq$ $\alpha$ for $g \in O(n) \backslash E$ and $\operatorname{dim} E \leq \alpha+n-1-\operatorname{dim} A-(n-1) \operatorname{dim} B / n+$ $(n-1)(n-2) / 2$. Moreover, $\operatorname{dim} E \leq \alpha+n-1-\operatorname{dim} A-\operatorname{dim} B+$ $(n-1)(n-2) / 2$, if $\operatorname{dim} B \leq(n-1) / 2$.

Notice that in some cases the upper bound for $\operatorname{dim} E$ is greater than $n-1+(n-1)(n-2) / 2=n(n-1) / 2=\operatorname{dim} O(n)$. Then we can take $E=$ $O(n)$ and the statement is empty.

Proofs of Theorems 4.2 and 4.3: The case $\operatorname{dim} A>(n+1) / 2$ in the first part of (1) of Theorem 4.2 follows from Lemma 13.9 in [29] or from Lemma 7.1 in $[\mathbf{3 0}]$. I am not aware of any exceptional set estimates under the condition $\operatorname{dim} A>(n+1) / 2$.

Let $0<s<\operatorname{dim} A$ and $0<t<\operatorname{dim} B$ and let $\mu \in \mathcal{M}(A), \nu \in \mathcal{M}(B)$ with $\mu(B(x, r)) \leq r^{s^{\prime}}, \nu(B(x, r)) \leq r^{t^{\prime}}$ for some $s^{\prime}>s, t^{\prime}>t$, and for $x \in \mathbb{R}^{n}, r>0$. Let $\lambda_{g}=S_{g \#}(\mu \times \nu) \in \mathcal{M}\left(S_{g}(A \times B)\right)$. Then $\widehat{\lambda_{g}}(\xi)=\widehat{\mu}(\xi) \widehat{\nu}\left(-g^{-1}(\xi)\right)$. For $0<\alpha \leq n$ we have by Lemma 4.1

$$
\begin{aligned}
\iint\left|\widehat{\lambda_{g}}(\xi)\right|^{2}|\xi|^{\alpha-n} d \xi d \theta g & =\int \sigma_{\theta}(\nu)(-\xi)|\widehat{\mu}(\xi)|^{2}|\xi|^{\alpha-n} d \xi \\
& \lesssim \int|\widehat{\mu}(\xi)|^{2}|\xi|^{\alpha-1-(n-1) t / n-\beta} d \xi \\
& =c I_{\alpha+n-1-(n-1) t / n-\beta}(\mu) \lesssim I_{s}(\mu)<\infty
\end{aligned}
$$

if $\beta \geq \alpha+n-1-(n-1) t / n-s$. 
Similarly, if $t \leq(n-1) / 2$,

$$
\iint\left|\widehat{\lambda_{g}}(\xi)\right|^{2}|\xi|^{\alpha-n} d \xi d \theta g \lesssim I_{\alpha+n-1-t-\beta}(\nu) \lesssim I_{s}(\mu)<\infty,
$$

if $\beta \geq \alpha+n-1-s-t$.

To get (1) of Theorems 4.2 and 4.3 we take $\alpha=n$. If $\beta \geq 2 n-1-$ $(n-1) t / n-s$, we have $S_{g \#}(\mu \times \nu) \ll \mathcal{L}^{n}$, and so $\mathcal{L}^{n}\left(S_{g}(A \times B)\right)>0$, for $\theta$ almost all $g \in O(n)$. In the case $\operatorname{dim} A+(n-1) \operatorname{dim} B / n>n$ we can choose $s$ and $t$ so that $n-1 \geq 2 n-1-(n-1) t / n-s$. Then we can take $\theta=\theta_{n}$ and $\beta=n-1$ to get (1) of Theorem 4.2 in this case. For Theorem 4.3(1) we have $\mathcal{L}^{n}\left(S_{g}(A \times B)\right)>0$ for $\theta$ almost all $g \in O(n)$ provided $\beta \geq 2 n-1-(n-1) t / n-s$. Using Proposition 3.2 we see from this that the set of $g \in O(n)$ for which $\mathcal{L}^{n}\left(S_{g}(A \times B)\right)=0$ has dimension at most $2 n-1-\operatorname{dim} A-(n-1) \operatorname{dim} B / n+(n-1)(n-2) / 2$. The case $\operatorname{dim} B \leq(n-1) / 2$ follows in the same way using (4.5).

For any $0<\alpha \leq n$ we have that if $\beta \geq \alpha+n-1-(n-1) t / n-s$, then by (4.4) $I_{\alpha}\left(S_{g \#}(\mu \times \nu)\right)<\infty$, and so $\operatorname{dim} S_{g}(A \times B) \geq \alpha$, for $\theta$ almost all $g \in O(n)$. To get the first statement of (2) of Theorem 4.2 we take $\alpha=(n-1) t / n+s$ and $\beta=n-1$. The case $\operatorname{dim} B \leq(n-1) / 2$ of Theorem 4.2(2) follows in the same way. For Theorem 4.3(2) we use Proposition 3.2 as before.

\section{Further discussion and open problems}

5.1. Averages over a cone. When $\theta=\theta_{n}$ we have for the integral in $(3.3)$

$$
\iint_{R \leq|\xi| \leq 2 R}|\widehat{\mu}(\xi, g(\xi))|^{2} d \xi d \theta_{n} g=R^{n} \int_{1 \leq|x|=|y| \leq 2}|\widehat{\mu}(R x, R y)|^{2} d \gamma(x, y),
$$

where the integration on the right side is with respect to a suitably normalized surface measure $\gamma$ on the conical surface $\Gamma=\left\{(x, y) \in \mathbb{R}^{n} \times\right.$ $\left.\mathbb{R}^{n}: 1 \leq|x|=|y| \leq 2\right\}$. Let $\phi$ be a smooth non-negative function with compact support in $\{y: 1 / 2<|y|<3\}$ and with $\phi(y)=1$ when $1 \leq|y| \leq 2$. Define the measure $\lambda$ by

$$
\int f d \lambda=\iint_{|x|=|y|} f(x, y) d \sigma_{|y|} x \phi(y) d y .
$$

Then $\gamma \lesssim \lambda$. 
The Fourier transform of $\lambda$ has the estimate

$$
|\widehat{\lambda}(\xi)| \lesssim|\xi|^{1-n}
$$

because

$$
\begin{aligned}
|\widehat{\lambda}(u, v)| & =\int e^{-2 \pi i(u \cdot x+v \cdot y)} d \lambda(x, y) \\
& =c \int e^{-2 \pi i v \cdot y \widehat{\sigma_{|y|}}(u) \phi(y) d y} \\
& =c \int e^{-2 \pi i v \cdot y}|y|^{n-1}|u|^{1-n \widehat{\sigma_{|u|}}}(y) \phi(y) d y \\
& =c|u|^{1-n} \mathcal{F}\left(|y|^{n-1} \phi(y) \widehat{\sigma_{|u|}}(y)\right)(v) \\
& =c|u|^{1-n} \int \mathcal{F}\left(|y|^{n-1} \phi(y)\right)(v-x) d \sigma_{|u|} x \approx|(u, v)|^{1-n},
\end{aligned}
$$

where the last estimate follows as for the Fourier transform of $\psi_{r}$ above. Let $\mu \in \mathcal{M}\left(\mathbb{R}^{2 n}\right)$ with $I_{s}(\mu)<\infty$. Then using a general theorem of Erdoğan, Theorem 1 in [7], we obtain for $R>1$

$$
\begin{aligned}
& \iint_{1 \leq|x|=|y| \leq 2}|\widehat{\mu}(R x, R y)|^{2} d \gamma(x, y) \\
& \quad \lesssim \iint|\widehat{\mu}(R x, R y)|^{2} d \lambda(x, y) \lesssim \begin{cases}R^{1-s} & \text { for all } 0<s<2 n \\
R^{-s} & \text { if } 0<s \leq n-1\end{cases}
\end{aligned}
$$

We shall not use these estimates in this paper. For Theorem 3.4 they give another proof for the almost all statements with respect to $\theta_{n}$, but they do not improve Theorem 3.4 and they do not give the exceptional set estimates. In fact, the second estimate in (5.2) is the same as (3.3) with $\theta=\theta_{n}, \beta=n-1$. Better decay estimates for (5.2) might lead to improvements for Theorem 3.4. In particular, any improvement of the exponent $1-s$ in the range $n<s<n+1$ would lead to an improvement of the first statement of Theorem 3.4(1). I am not aware of such results. However, in addition to the spherical averages (discussed in Section 4), which have been studied for a long time, there are recent estimates for cones and hyperboloids; see [2], [13], and [1].

The estimates (5.2) can be improved for product measures. We just plug in the spherical estimates from (4.2). Let $\mu, \nu \in \mathcal{M}\left(\mathbb{R}^{n}\right)$ be such 
that for some $0<s \leq n$ and $0<t \leq n$ we have $\mu(B(x, r)) \leq r^{s}$ and $\nu(B(x, r)) \leq r^{t}$ for $x \in \mathbb{R}^{n}, r>0$. Then for all $\epsilon>0, R>1$

$$
\begin{aligned}
\iint_{1 \leq|x|=|y| \leq 2} \mid \widehat{\mu \times \nu}(R x, & R y)\left.\right|^{2} d \gamma(x, y) \\
& \lesssim \begin{cases}R^{-s-(n-1) t / n+\epsilon} & \text { if } 0<s<n \\
R^{-s-t+\epsilon} & \text { if } 0<t \leq(n-1) / 2\end{cases}
\end{aligned}
$$

To see this, note that $\widehat{\mu \times \nu}(x, y)=\widehat{\mu}(x) \widehat{\nu}(y)$. Then if $\sigma(\nu)(r) \lesssim r^{-\alpha}$, we get by $(3.5)$

$$
\begin{aligned}
\iint_{1 \leq|x|=|y| \leq 2}|\widehat{\mu \times \nu}(R x, R y)|^{2} d \gamma(x, y) & =c \int_{1 \leq|x| \leq 2} \sigma(\nu)(R|x|)|\widehat{\mu}(R x)|^{2} d x \\
& \lesssim R^{-\alpha} \int_{1 \leq|x| \leq 2}|\widehat{\mu}(R x)|^{2} d x \lesssim R^{-\alpha-s},
\end{aligned}
$$

and the claims follow from (4.2).

5.2. Distance sets and measures. There are some connections between this topic and Falconer's distance set problem. For general discussion and references, see for example [30]. Falconer showed in [10] that for a Borel set $A \subset \mathbb{R}^{n}$ the distance set $\{|x-y|: x, y \in A\}$ has positive Lebesgue measure if $\operatorname{dim} A>(n+1) / 2$. We had the same condition in Theorem 4.2 and it appeared in the intersection results of [27]. When $n=2$ Wolff $([\mathbf{3 8}])$ improved $3 / 2$ to $4 / 3$. Observe that when $\operatorname{dim} A=\operatorname{dim} B$, the assumption $\operatorname{dim} A+\operatorname{dim} B / 2>2$ in Theorem 4.2 becomes $\operatorname{dim} A>4 / 3$ and is the same as Wolff's. In [15] Iosevich and Liu improved distance set results of the time for product sets with rather simple arguments. For the most recent, and so far the best-known, distance set results, see $[\mathbf{1 2}],[\mathbf{4}]$, and [5].

The proofs of distance set results often involve the distance measure $\delta(\mu)$ of a measure $\mu$ defined by

$$
\delta(\mu)(B)=\mu \times \mu(\{(x, y):|x-y| \in B\}), \quad B \subset \mathbb{R} .
$$

For example, Wolff showed that $\delta(\mu) \in L^{2}(\mathbb{R})$, if $I_{s}(\mu)<\infty$ for some $s>4 / 3$. To do this he used decay estimates for the spherical averages $\sigma(\mu)(r)$ and proved (4.2) for $n=2$. 
From the argument in Subsection 3.2 we see that when $\mu$ is replaced by $\mu \times \nu$ we have

$$
\begin{aligned}
& \iint \underline{D}\left(S_{g \#}(\mu \times \nu)\right)(z)^{2} d z d \theta_{n} g \\
& \quad \leq \liminf _{r \rightarrow 0} \iint \alpha(n)^{-1} r^{-n} S_{g \#}(\mu \times \nu)(B(z, r)) d S_{g \#}(\mu \times \nu) z d \theta_{n} g \\
& =\liminf _{r \rightarrow 0} \int \alpha(n)^{-1} r^{-n} \theta_{n}(\{g:|x-g(y)-(u-g(v))| \leq r\}) \\
& \quad d(\mu \times \nu)(x, y) d(\mu \times \nu)(u, v) \\
& \leq \liminf _{r \rightarrow 0} c \int r^{-1} \mu \times \mu(\{(x, u):|| x-u|-| y-v|| \leq r\})|y-v|^{1-n} \\
& =\liminf _{r \rightarrow 0} \int r^{-1} \delta(\mu)(B(t, r)) t^{1-n} d \delta(\nu) t \quad d(\nu \times \nu)(y, v) \\
& =c \int \delta(\mu)(t) \delta(\nu)(t) t^{1-n} d t,
\end{aligned}
$$

provided the distance measures $\delta(\mu)$ and $\delta(\nu)$ are $L^{2}$ functions, and even a bit better so that we can move liminf inside the integral. In fact, we have equality everywhere in the above argument if $\mu$ and $\nu$ are smooth functions with compact support. Since by an example in [12], when $n=2$, for any $s<4 / 3, I_{s}(\mu)<\infty$ is not enough for $\delta(\mu)$ to be in $L^{2}$, probably it is not enough for $S_{g \#}(\mu \times \mu)$ to be in $L^{2}$. But in [12] it was shown that if $I_{s}(\mu)<\infty$ for some $s>5 / 4$, there is a modification of $\mu$ with good $L^{2}$ behaviour. Maybe this method could be used to show, for instance, that if $n=2$ and $\operatorname{dim} A=\operatorname{dim} B>5 / 4$, then $\mathcal{L}^{2}\left(\mathcal{S}_{g}(A \times\right.$ $B))>0$ for almost all $g \in O(2)$. One problem is that for distance sets one can split the measure into two parts with positive distance and only consider distances between points in the different supports, so one need not consider arbitrarily small distances, and the authors of [12] seem to use this essentially. Here such reduction may not be possible.

5.3. Hausdorff dimension of intersections. One motivation for this study is to shed light on intersection problems. The main question is: What conditions on $\operatorname{dim} A$ and $\operatorname{dim} B$ guarantee that for almost all $g \in$ $O(n), \operatorname{dim} A \cap(g(B)+z) \geq \operatorname{dim} A+\operatorname{dim} B-n-\epsilon$ for positively many $z \in$ $\mathbb{R}^{n}$ for every $\epsilon>0$ ? I expect that $\operatorname{dim} A+\operatorname{dim} B>n$ should be enough. This is only known when one of the sets has dimension greater than $(n+$ 1)/2. A necessary condition, of course, is that $\mathcal{L}^{n}\left(S_{g}(A \times B)\right)>0$ for almost all $g \in O(n)$. By Theorem 4.2 we have this when $\operatorname{dim} A+(n-$ 
1) $\operatorname{dim} B / n>n$, but even then for general $A$ and $B$ I only know the estimate $\operatorname{dim} A \cap(g(B)+z) \geq \operatorname{dim} A+(n-1) \operatorname{dim} B / n-n-\epsilon$, which follows from Theorem 4.1 in $[\mathbf{3 1}]$ and (4.2). Since $A \cap(g(B)+z)$ is the projection on the first factor of $(A \times B) \cap S_{g}^{-1}(z)$, the problem is equivalent to getting dimension estimates for the sections $(A \times B) \cap$ $S_{g}^{-1}(z)$. This point of view together with the results of Section 4 is used in [33] to show that for almost all $g \in O(n), \operatorname{dim} A \cap(g(B)+z) \geq$ $\operatorname{dim} A+\operatorname{dim} B-n$ for positively many $z \in \mathbb{R}^{n}$ if $A$ and $B$ have positive and finite Hausdorff measures in their dimensions and $\liminf _{r \rightarrow 0} r^{-s} \mathcal{H}^{s}(A \cap$ $B(x, r))>0$ for $\mathcal{H}^{s}$ almost all $x \in A$, where $s=\operatorname{dim} A$, and respectively for $B$.

Replacing $O(n)$ with the larger group of similarities, maps $r g, r>0$, $g \in O(n)$, better results were obtained in [22], [26], and [31]. Then no condition like $\operatorname{dim} A>(n+1) / 2$ is needed. In fact, Kahane considered the more general situation of closed subgroups of the general linear group which act transitively outside the origin.

\section{References}

[1] A. Barron, M. B. ErdoĞan, and T. L. J. Harris, Fourier decay of fractal measures on hyperboloids, Trans. Amer. Math. Soc. 374(2) (2021), 1041-1075. DOI : $10.1090 / \operatorname{tran} / 8283$.

[2] C.-H. Cho, S. Ham, And S. Lee, Fractal Strichartz estimate for the wave equation, Nonlinear Anal. 150 (2017), 61-75. DOI: 10.1016/j.na.2016.11.006.

[3] X. Du, Upper bounds for Fourier decay rates of fractal measures, J. Lond. Math. Soc. (2) 102(3) (2020), 1318-1336. DOI : 10.1112/jlms.12364.

[4] X. Du, L. Guth, Y. Ou, H. Wang, B. Wilson, and R. Zhang, Weighted restriction estimates and application to Falconer distance set problem, Amer. J. Math. 143(1) (2021), 175-211. DOI : 10.1353/ajm.2021.0005.

[5] X. Du, A. Iosevich, Y. Ou, H. Wang, and R. Zhang, An improved result for Falconer's distance set problem in even dimensions, Math. Ann. 380(3-4) (2021), 1215-1231. DOI : 10.1007/s00208-021-02170-1.

[6] X. Du AND R. Zhang, Sharp $L^{2}$ estimates of the Schrödinger maximal function in higher dimensions, Ann. of Math. (2) 189(3) (2019), 837-861. DOI : 10.4007/ annals.2019.189.3.4.

[7] M. B. ERdoĞan, A note on the Fourier transform of fractal measures, Math. Res. Lett. 11(2-3) (2004), 299-313. DOI : 10.4310/MRL.2004.v11.n3.a3.

[8] S. Eswarathasan, A. Iosevich, and K. Taylor, Intersections of sets and Fourier analysis, J. Anal. Math. 128 (2016), 159-178. DOI : 10.1007/s11854-016 $-0004-1$.

[9] K. J. FAlConer, Hausdorff dimension and the exceptional set of projections, Mathematika 29(1) (1982), 109-115. DOI: 10.1112/S0025579300012201.

[10] K. J. Falconer, On the Hausdorff dimensions of distance sets, Mathematika 32(2) (1985), 206-212 (1986). DOI : 10.1112/S0025579300010998.

[11] K. Fässler and T. Orponen, On restricted families of projections in $\mathbb{R}^{3}$, Proc. Lond. Math. Soc. (3) 109(2) (2014), 353-381. DOI : 10.1112/plms/pdu004. 
[12] L. Guth, A. Iosevich, Y. Ou, and H. Wang, On Falconer's distance set problem in the plane, Invent. Math. 219(3) (2020), 779-830. DOI: 10.1007/ s00222-019-00917-x.

[13] T. L. J. HARRIS, Improved decay of conical averages of the Fourier transform, Proc. Amer. Math. Soc. 147(11) (2019), 4781-4796. DOI : 10.1090/proc/14747.

[14] T. L. J. HARRIS, Restricted families of projections onto planes: the general case of nonvanishing geodesic curvature, Preprint (2021). arXiv:2107.14701.

[15] A. Iosevich AND B. LiU, Falconer distance problem, additive energy and Cartesian products, Ann. Acad. Sci. Fenn. Math. 41(2) (2016), 579-585. DOI : $10.5186 /$ aasfm. 2016.4135.

[16] E. Järvenpä̈̈, M. JÄrvenpäÄ, And T. Keleti, Hausdorff dimension and nondegenerate families of projections, J. Geom. Anal. 24(4) (2014), 2020-2034. DOI : $10.1007 / \mathrm{s} 12220-013-9407-8$.

[17] E. JärvenpäÄ, M. JärvenpäÄ, F. Ledrappier, and M. Leikas, One-dimensional families of projections, Nonlinearity 21(3) (2008), 453-463. DOI : 10.1088/ 0951-7715/21/3/005.

[18] M. JÄRVENPÄ̈̈, On the upper Minkowski dimension, the packing dimension, and orthogonal projections, Ann. Acad. Sci. Fenn. Ser. A I Math. Dissertationes 99 (1994), $34 \mathrm{pp}$.

[19] M. JÄRVEnPÄ̈̈, Concerning the packing dimension of intersection measures, Math. Proc. Cambridge Philos. Soc. 121(2) (1997), 287-296. DOI: 10.1017/ S0305004196001417.

[20] M. JÄrVEnPÄÄ, Packing dimension, intersection measures, and isometries, Math. Proc. Cambridge Philos. Soc. 122(3) (1997), 483-490. DOI: 10.1017/ S0305004197001941.

[21] A. Käenmäki, T. Orponen, And L. Venieri, A Marstrand-type restricted projection theorem in $\mathbb{R}^{3}$, Preprint (2017). arXiv:1708.04859.

[22] J.-P. Kahane, Sur la dimension des intersections, in: "Aspects of Mathematics and its Applications", North-Holland Math. Library 34, North-Holland, Amsterdam, 1986, pp. 419-430. DOI : 10.1016/S0924-6509(09) 70272-7.

[23] R. Kaufman, On Hausdorff dimension of projections, Mathematika 15(2) (1968), 153-155. DOI : 10.1112/S0025579300002503.

[24] R. Kaufman and P. Mattila, Hausdorff dimension and exceptional sets of linear transformations, Ann. Acad. Sci. Fenn. Ser. A I Math. 1(2) (1975), 387-392. DOI : $10.5186 /$ AASFM. 1975.0105.

[25] J. M. Marstrand, Some fundamental geometrical properties of plane sets of fractional dimensions, Proc. London Math. Soc. (3) 4(1) (1954), 257-302. DOI : $10.1112 / \mathrm{plms} / \mathrm{s} 3-4.1 .257$.

[26] P. Mattila, Hausdorff dimension and capacities of intersections of sets in $n$ space, Acta Math. 152(1-2) (1984), 77-105. DOI : 10.1007/BF02392192.

[27] P. Mattila, On the Hausdorff dimension and capacities of intersections, Mathematika 32(2) (1985), 213-217 (1986). DOI : 10.1112/S0025579300011001.

[28] P. Mattila, Spherical averages of Fourier transforms of measures with finite energy; dimension of intersections and distance sets, Mathematika 34(2) (1987), 207-228. DOI : 10.1112/S0025579300013462.

[29] P. Mattila, "Geometry of Sets and Measures in Euclidean Spaces", Fractals and rectifiability, Cambridge Studies in Advanced Mathematics 44, Cambridge University Press, Cambridge, 1995. DOI : 10.1017/CB09780511623813. 
[30] P. Mattila, "Fourier Analysis and Hausdorff Dimension", Cambridge Studies in Advanced Mathematics 150, Cambridge University Press, Cambridge, 2015. DOI : $10.1017 /$ CB09781316227619.

[31] P. Mattila, Exceptional set estimates for the Hausdorff dimension of intersections, Ann. Acad. Sci. Fenn. Math. 42(2) (2017), 611-620. DOI: 10.5186/aasfm. 2017.4236.

[32] P. Mattila, Hausdorff Dimension, Projections, Intersections, and Besicovitch Sets, in: "New Trends in Applied Harmonic Analysis", Volume 2, Applied and Numerical Harmonic Analysis, Birkhäuser, Cham, 2019, pp. 129-157. DOI : 10. 1007/978-3-030-32353-0_6.

[33] P. Mattila, Hausdorff dimension of intersections with planes and general sets, Preprint (2020). arXiv:2005.11790.

[34] D. M. OberLin, Exceptional sets of projections, unions of $k$-planes and associated transforms, Israel J. Math. 202(1) (2014), 331-342. DOI: 10.1007/ s11856-014-1040-4.

[35] D. Oberlin and R. Oberlin, Application of a Fourier restriction theorem to certain families of projections in $\mathbb{R}^{3}$, J. Geom. Anal. 25(3) (2015), 1476-1491. DOI : $10.1007 / \mathrm{s} 12220-014-9480-7$.

[36] T. OrPonen, Hausdorff dimension estimates for restricted families of projections in $\mathbb{R}^{3}$, Adv. Math. 275 (2015), 147-183. DOI : 10.1016/j.aim.2015.02.011.

[37] T. ORPONEN AND L. Venieri, Improved bounds for restricted families of projections to planes in $\mathbb{R}^{3}$, Int. Math. Res. Not. IMRN 2020(19) (2020), 5797-5813. DOI : $10.1093 /$ imrn/rny193.

[38] T. WolfF, Decay of circular means of Fourier transforms of measures, Internat. Math. Res. Notices 1999(10) (1999), 547-567. DOI : 10.1155/S1073792899 000288.

Department of Mathematics and Statistics, P.O. Box 68, FI-00014 University of Helsinki, Finland

E-mail address: pertti.mattila@helsinki.fi

Received on May 26, 2020.

Accepted on September 22, 2020. 